\title{
Determination of source spectra of ultrahigh energy cosmic rays
}

\author{
V.S. Ptuskin, S.I. Rogovaya* and V.N. Zirakashvili \\ Pushkov Institute of Terrestrial Magnetism, Ionosphere and Radio Wave Propagation of the \\ Russian Academy of Sciences (IZMIRAN), Troitsk, Moscow 142190, Russia \\ E-mail: vptuskin@izmiran.ru, rogovaya@izmiran.ru, zirakeizmiran.ru
}

\begin{abstract}
The energy spectra and composition of ultra-high energy cosmic rays are changing in a course of propagation in the expanding Universe filled with background radiation. We use a numerical code for solution of inverse problem for cosmic-ray transport equations that enables the determination of source spectra of different nuclei from the cosmic ray spectra observed at the Earth. The source spectrum is calculated without ad hoc assumptions about its' shape. The spectra of protons and iron nuclei injected from the extragalactic sources are found. The effects of cosmological source evolution and the finite distance to the nearest source are studied. The data from the Auger experiment are used to illustrate the method.
\end{abstract}

The 34th International Cosmic Ray Conference,

30 July- 6 August, 2015

The Hague, The Netherlands

${ }^{*}$ Speaker. 


\section{Introduction}

The energy spectrum in extragalactic sources is commonly determined by the trial-and-error method when one makes the calculations of the expected at the Earth cosmic ray intensity assuming some shape of the source energy spectrum and the source composition. The calculations follow cosmic ray propagation from the source to the observer, e. g. [1]. The standard assumption is that the source spectrum is a power law on magnetic rigidity up to some maximum rigidity.

In the present work we show how to inverse the procedure and calculate the source function starting from the observed at the Earth spectrum without ad hoc assumptions about the shape of source spectrum. Simple cases of the source composition that includes protons and Iron nuclei are considered and the analytical approximations of the data from Auger experiment is used. The approach of inverse transport problem allows the abandonment of the standard assumption of power law source spectrum with an abrupt cutoff at some maximum magnetic rigidity as it is usually assumed when the direct problem is considered.

\section{Transport equations}

We use the following transport equation for cosmic ray protons and nuclei in the expanding Universe filled with the background electromagnetic radiation (see [2] for detail):

$$
\begin{array}{r}
-H(z)(1+z) \frac{\partial}{\partial z}\left(\frac{F(A, \varepsilon, z)}{(1+z)^{3}}\right)- \\
-\frac{\partial}{\partial \varepsilon}\left(\varepsilon\left(\frac{H(z)}{(1+z)^{3}}+\frac{1}{\tau(A, \varepsilon, z)}\right) F(A, \varepsilon, z)\right)+v(A, \varepsilon, z) F(A, \varepsilon, z) \\
=\sum_{i=1,2 \ldots} v(A+i \rightarrow A, \varepsilon, z) F(A+i, \varepsilon, z)+q(A, \varepsilon)(1+z)^{m} .
\end{array}
$$

The system of eqs. (2.1) for all kinds of nuclei with different mass numbers $A$ from Iron to Hydrogen should be solved simultaneously. The energy per nucleon $\varepsilon=E / A$ is used here because it is approximately conserved in a process of nuclear photodisintegration, $F(A, \varepsilon, z)$ is the corresponding cosmic-ray distribution function, $z$ is the redshift, $q(A, \varepsilon)$ is the density of cosmicray sources at the present epoch $z=0, m$ characterizes the source evolution (the evolution is absent for $m=0), \tau(A, \varepsilon, z)$ is the characteristic time of energy loss by the production of $e^{-} e^{+}$pairs and pions, $v(A, \varepsilon, z)$ is the frequency of nuclear photodisintegration, the sum in the right side of eq. (2.1) describes the contribution of secondary nuclei produced by the photodisintegration of heavier nuclei, $H(z)=H_{0}\left((1+z)^{3} \Omega_{m}+\Omega_{\Lambda}\right)^{1 / 2}$ is the Hubble parameter in a flat universe with the matter density $\Omega_{m}(=0.3)$ and the $\Lambda$-term $\Omega_{\Lambda}(=0.7)$.

The numerical solution of cosmic-ray transport equations follows the finite differences method. The variables are the redshift $z$ and $\log (E / A)$.

Let us introduce solution $G\left(A, \varepsilon ; A_{s}, \varepsilon_{s}\right)$ of eqs. (2.1) at $z=0$ for a delta-source $q(A, \varepsilon)=$ $\delta_{A A_{s}} \delta\left(\varepsilon-\varepsilon_{s}\right)$. This source function describes the emission of nuclei with mass number $A_{s}$ and energy $\varepsilon_{s}$ from cosmic ray sources distributed over all $z$ up to some $z$ max. The general solution of 


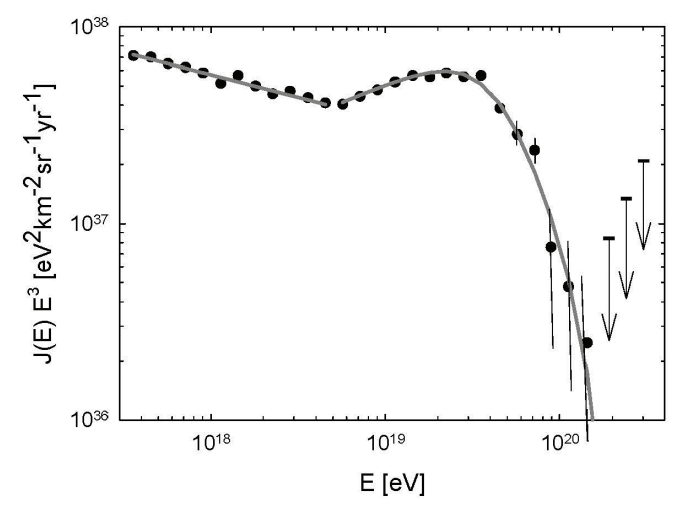

Figure 1: Analytical approximations used in the present calculations to describe the Auger data are shown by solid line together with Auger data [3].

eqs. (2.1) at the observer location $z=0$ can now be presented as

$$
F(A, \varepsilon, z=0)=\sum_{A^{\prime}} \int d \varepsilon^{\prime} G\left(A, \varepsilon ; A^{\prime}, \varepsilon^{\prime}\right) q\left(A^{\prime}, \varepsilon^{\prime}\right) .
$$

The observed all-particle spectrum is determined by the summation over all types of nuclei $\sum_{A} F(A, \varepsilon, z=$ $0)$.

Eqs. for $F$ should be presented in the matrix form and inverted to find the source functions $q(A, \varepsilon)$ for different types of nuclei. This procedure is described at length in [4].

To simplify calculations and damp the spread of data points in the measured at the Earth cosmic ray spectrum, we use its analytical approximation.

The formula

$$
\begin{array}{r}
J(E) \propto E^{-3.23}, E<5 \times 10^{18} \mathrm{eV} \\
J(E) \propto E^{-2.63} \times\left[1+\exp \left(\log \left(E / 10^{19.63} \mathrm{eV}\right) / 0.15\right)\right]^{-1} \times \\
\exp \left(-\left(E /\left(1.5 \times 10^{20} \mathrm{eV}\right)\right)^{4}\right), E>5 \times 10^{18} \mathrm{eV} .
\end{array}
$$

is used in the calculations to approximate the Auger data [3], see figure 1. This formula is similar to the equation suggested by the Auger team but contains $\exp \left(-\left(E / 1.5 \times 10^{20} \mathrm{eV}\right)^{4}\right)$ factor of $\operatorname{cosmic}$ ray flux suppression at energies $\gtrsim 1.5 \times 10^{20} \mathrm{eV}$.

Using the Auger data on energy dependence of the mean logarithm of the atomic mass number $\langle\ln A\rangle$ calculated in the EPOS-LHC model of particle interactions in the atmosphere [3], we accept the following approximation

$$
\langle\ln A\rangle=0.5+4.2 \times\left(E / 10^{20} \mathrm{eV}\right)^{0.6}
$$

shown by the dash line in figure 5 below. 


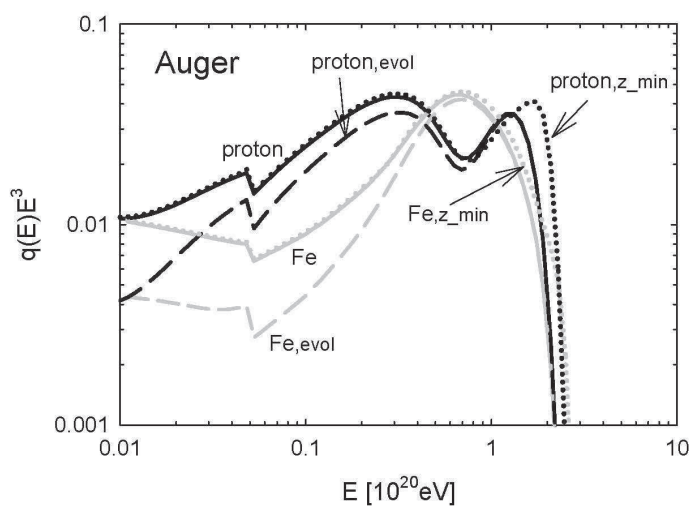

Figure 2: Calculated source spectra in arbitrary units based on the approximated analytically Auger data. Black lines for proton source; gray lines for Iron source. Solid lines correspond to the homogeneous source distribution without evolution, $m=0$. Dotted lines correspond to the spatial source distribution with a finite distance to the nearest source located at the redshift $z_{\min }=0.0024$ at $m=0$. Dash lines are the source spectra for homogeneous source distribution with evolution described in the text.

\section{Results of Calculations}

We first make calculations of the source spectra in two simple cases of pure proton and pure Iron source composition. The results are shown in figure 2. The dark line refer to a pure proton source and the gray line refer to a pure Iron source. The solid lines illustrate the case without source evolution $m=0$; the dash lines describe the case of AGN with a strong evolution where $m=3.2$ at $z<1.2$ and the evolution is saturated at larger $z$ [6]. It is clear from figure 2 that strong cosmological evolution leads to the decrease of required source power at low cosmic ray energies. The difference with the source spectrum without evolution reaches the factor of about 4 at $10^{18}$ $\mathrm{eV}$. The dotted line show the results of calculations with a non-zero distance to the nearest source $z_{\min } \neq 0$ for the source number density $n_{s}=10^{-4} \mathrm{Mpc}^{-3}$. It is clear that the finite distance to the nearest source requires the increase of source power at the highest energies of accelerated particles.

The kinks in all source spectra at about $5 \times 10^{18} \mathrm{eV}$ are due to the corresponding discontinuities of the first derivatives of the expression (2.3) and in this sense they are artificial. The difference between proton and Iron sources at low energies is evident: the proton source spectrum can be better approximated by a power law compared to the concave Iron spectrum. It is explained by the influence of energy loss on the $e^{-} e^{+}$production in the case of ultra high energy protons moving through the background radiation [5]. After propagation in the intergalactic space, this process produces the characteristic dip in the initial power law proton spectrum at around $5 \times 10^{18} \mathrm{eV}$ while the Iron nuclei preserve the shape of their source spectrum.

The results of calculations when both proton and Iron are present in the source and their spectra are similar functions of magnetic rigidity are shown in figure 3 . One can see how the results of calculations depend on the assumed Iron-to-proton source ratio $S_{F e} / S_{p}$. 


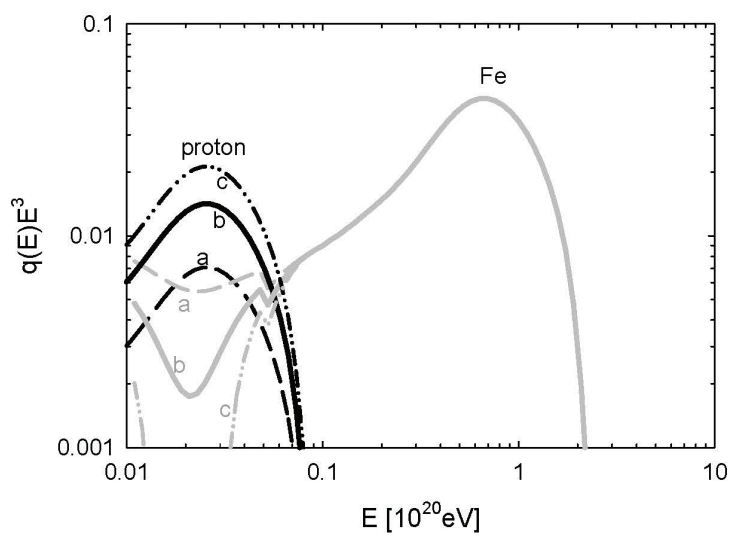

Figure 3: Calculated source spectra based on the Auger data for different Iron-to-proton source ratios indicated by (a) for $S_{F e} / S_{p}=2 \times 10^{-2}$, (b) for $S_{F e} / S_{p}=10^{-2}$, and (c) for $S_{F e} / S_{p}=6.7 \times 10^{-3}$. Black lines for proton source; gray lines for Iron source. Proton and Iron source spectra have the same dependence on magnetic rigidity. Homogeneous source distribution without evolution is assumed.

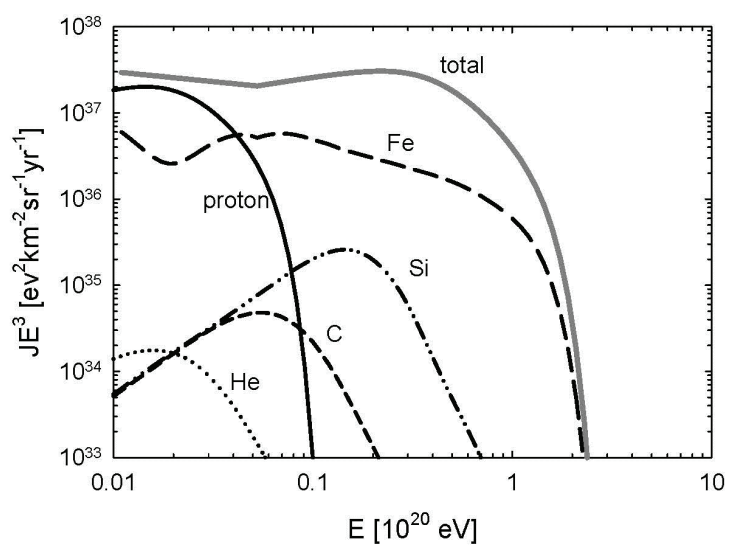

Figure 4: Calculated spectra of different types of nuclei for Iron-to-proton source ratio $S_{F e} / S_{p}=10^{-2}$ and the total Auger cosmic ray spectrum at the Earth.

The calculated elemental composition of cosmic rays at the Earth for the case $S_{F e} / S_{p}=10^{-2}$ is presented in figure 4. The corresponding value of $\langle\ln (A)\rangle$ is shown in figure 5. It is evident that our very simple model with only two primary species at the source (protons and Iron nuclei), does not reproduce the observed $\langle\ln A\rangle$ except the energies $\sim 10^{18} \mathrm{eV}$ where the protons and light nuclei dominate and the highest energies where the Iron group nuclei dominate. The intermediate nuclei are certainly needed at the source to reproduce observations at all energies.

The source spectra of protons and Iron can be found if the measurements of the mean logarithm 


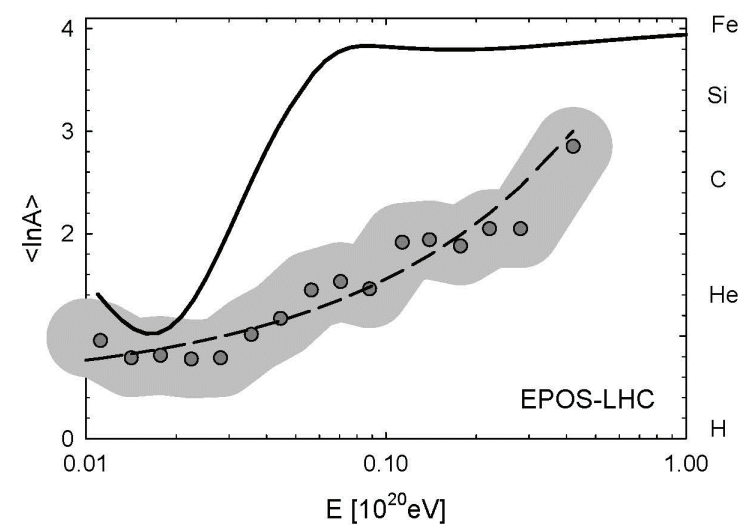

Figure 5: Calculated value of $\langle\ln (A)\rangle$ (solid line) together with corresponding Auger data (dots and gray regions which characterizes errors in determination of $\langle\ln (A)\rangle$ in the EPOS LHC interaction model). Dash line shows our approximation (2.4).

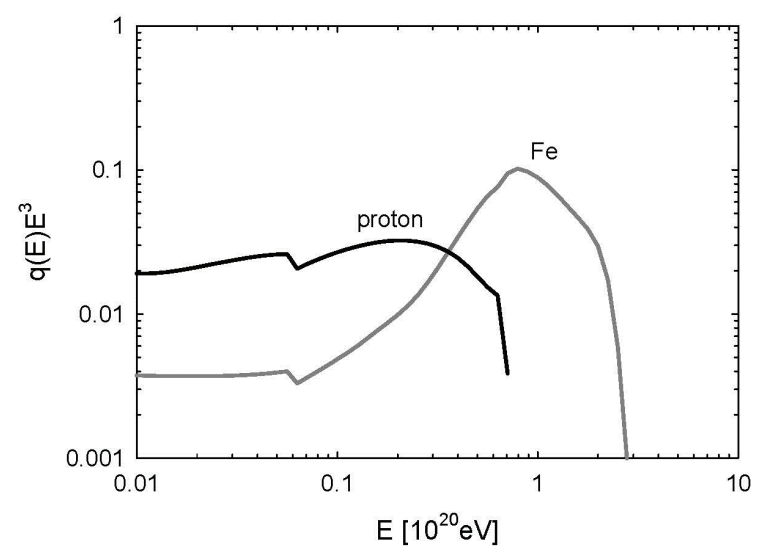

Figure 6: Calculated source spectra of protons and Iron based on Auger data on cosmic ray spectrum and $\langle\ln A\rangle$. Homogeneous source distribution without evolution is assumed.

$\langle\ln A\rangle$ are available in addition to the all-particle spectrum.

Both, the observed all particle cosmic-ray Auger spectrum at the Earth approximated by eq. (2.3) and the $\langle\ln A(E)\rangle$ given by eq. (2.4) can be exactly reproduced assuming that only protons and Iron nuclei are present in the source. The corresponding calculated source spectra are shown in figure 6 . They have different dependence on magnetic rigidity. 


\section{Discussion and Conclusion}

We showed how one can find average spectrum of extragalactic sources from the cosmic ray spectrum observed at the Earth. This task was formulated as an inverse problem for the system of transport eqs. (2.1) that describe the propagation of ultra-high energy cosmic rays in the expanding Universe filled with the background electromagnetic radiation. The simple settings were considered. The Auger data [3] were approximated by formula (2.3). It was assumed that only two kinds of nuclei (proton and Iron) were present in the sources and the cases of a pure proton, pure Iron, and the mixed source composition were considered. In the last case the calculations were made for the following two scenarios: 1) the proton and Iron source spectra have the same shape on rigidity; 2) the source spectra and the Iron-to-proton ratio are forced to reproduce the observed at the Earth value of $\langle\ln A\rangle$. Mathematically, the inverse problems for transport equations (2.1) are ill-posed in the general case that manifests itself in the instability of the derived numerical solutions. It explains to a large extent simple assumptions used in the present paper. We plan specifically study this problem in a future publication.

Two additional factors that may impact on the interpretation of the derived source spectra at energies close to $10^{18} \mathrm{eV}$ is worth to mention. The first is the possible contribution of the Galactic sources that may dominate in the observed cosmic ray spectrum at $<3 \times 10^{18} \mathrm{eV}$, see e.g. [5] for discussion. The second is the strong deflection of cosmic ray trajectories in magnetic field that may produce the so called magnetic horizon effect in the expanding Universe. The particle transmission factor at various distances to the source as a function of particle energy was calculated in [7]. This factor characterises the suppression of cosmic ray intensity due to the magnetic horizon effect. Using results of these calculations and assuming that the value of the intergalactic magnetic field is $1 \mathrm{nG}$ and its correlation length is $1 \mathrm{Mpc}$, one can find that the magnetic horizon effect is not significant for Iron nuclei with energies above $10^{18} \mathrm{eV}$ if cosmic ray source density is not smaller than $\sim 10^{-4} \mathrm{Mpc}^{-3}$. It is in the limits of the low bound on cosmic ray sources density found at the GZK energies in the Auger experiment [8]. It should be pointed out that the source density may increase with the decreasing of energy of accelerated particles. For example, such a behavior was found in our model of cosmic ray acceleration by the AGN jets with the observed distribution on kinetic energy where more numerous weak jets contribute most to small cosmic ray energies [2]. The experimental indication of this effect was found in [9]. The calculations of the source spectra in the present paper ignored the presence of the intergalactic magnetic field.

It is difficult to make firm astrophysical statements about cosmic ray source spectra and composition from our simple modelling. However, some conclusions can be made. Recall that the kinks in the calculated source spectra at about $5 \times 10^{18} \mathrm{eV}$ reflect the corresponding discontinuities in the derivatives of the approximation eq. (2.3) and are artificial in this sense. To demonstrate the specific character of the inverse problem solutions, we did not correct the unphysical approximations of the observed spectra. The Auger data favor the transition from a proton source composition to the Iron one as the energy is rising. With our simple two-species composition, this case is most closely reproduced by the calculations illustrated in figure 3 . The obtained source spectra resemble the results $[10,11]$ based on the analysis of direct transport problems with a power law source spectrum. The maximum magnetic rigidity of accelerated particles $(3 \ldots .5) \times 10^{18} \mathrm{eV}$ is relatively low in this case that alleviates the problem of cosmic ray acceleration to the extremely high energies. The 
calculated composition of cosmic rays at the Earth shown in figure 5 considerably deviates from the Auger measurements and certainly requires incorporation of the intermediate nuclei between protons and Iron in the source composition. More detailed presentation of our work can be found in [4].

\section{Acknowledgments}

The work was supported by the Russian Foundation for Basic Research grant 13-02-00056 and by the Russian Federation Ministry of Science and Education contract 14.518.11.7046. The work was partly fulfilled during VSP visit to the University of Maryland where it was supported by the NASA grant NNX13AC46G.

\section{References}

[1] D. Allard, Propagation of extragalactic ultra-high energy cosmic-ray nuclei: implications for the observed spectrum and composition, eprint arXiv:0906.3156v1 (2009)

[2] V.S. Ptuskin, S.I. Rogovaya and V.N. Zirakashvili, On ultra-high energy cosmic rays: origin in AGN jets and transport in expanding Universe, Adv. Space Res. 51 (2013) 315

[3] A. Letessier-Selvon for the Pierre Auger Collaboration, Highlights from the Pierre Auger Observatory 33rd International Cosmic Ray Conference, Rio de Janeiro, 2013, in press eprint arXiv:1310.2118v1 (2013)

[4] V.S. Ptuskin, S.I. Rogovaya and V.N. Zirakashvili, Inverse problem for extragalactic transport of ultra-high energy cosmic rays, JCAP 03 (2015) 054

[5] V.S. Berezinsky, A. Gazizov and S. Grigorieva, On astrophysical solution to ultrahigh energy cosmic rays, Phys. Rev. D 74 (2006) 3005

[6] A.J. Barger, L.L. Cowie, Mushotzky et. al. The cosmic evolution of hard X-ray-selected Active Galactic Nuclei, Astron J 129 (2005) 578

[7] K. Kotera, M. Lemoine, Inhomogeneous extragalactic magnetic fields and the second knee in the cosmic ray spectrum, Phys Rev D 77 (2008) 3005

[8] The Pierre Auger collaboration, Bounds on the density of sources of ultra-high energy cosmic rays from the Pierre Auger Observatory, JCAP 05 (2013) 009

[9] H. Takami, K. Sato, Astropart Phys, 30 (2009) 306

[10] D. Allard, N.G.Busca, G.Decerprit, A.V.Olinto, E.Parizot, Implications of the cosmic ray spectrum for the mass composition at the highest energies, J. Cosmol. Astropart.Phys. 10 (2008) 33

[11] R. Aloisio, V.Berezinsky, A.Gazizov, Ultra high energy cosmic rays: the disappointing model Astropart. Phys. 34 (2011) 620 